vulsed. At 11 A.M. a fit commencing at the left angle of the mouth passed to the muscles on the left side of the face, and after continuing for a minute passed to the muscles of the other side of the face. The spasms were clonic, and more violent on the left side than on the right. The pupils, semi-contracted, reacted to light. Consciousness was not lost. A few minutes later clonic spasms came on in the abdominal muscles, chiefly on the left side, but affecting the right to a slight extent. The boy was at this time lying on his back in a drowsy state; but he would put out his tongue when ordered to do so, though he resented interference as he had done before the operation. He moved the left arm very slightly, and also the left leg. The prick of a pin was felt equally on the two sides; the knee-jerks were very slight on either side, though more marked on the right. Temperature $102 \cdot 4^{\circ}$; pulse 112 ; respiration 24 . The wound was dressed, and the scalp was found to be puffy for some distance around the wound. Tongue covered with white fur. Bowels not open. Taking food regularly. In the evening the temperature was $1024^{\circ}$; pulse 136 , regular, weak, compressible. At 1.45 P.M. he had a fit, only lasting a minute or two, involving the left side of the face and left arm. On June 17 th, when seen at noon, there had been no recurrence of fits. He was sensible; the pupils equal, and responding to light. There were much stronger and freer movements of the left leg; those of the left arm were slight, but freer than on the previous day. He was drowsy. The pulse was less compressible and fuller; temperature 101. The tongue was coated but moist. An enema had been followed by a free evacuation. The wound was dressed. There was less puffiness of the scalp, the granulations were healthy, there was less discharge, and the pus was healthy and free from smell. On the 18 th the patient, though still inclined to sleep, was quite sensible when roused, but irritable, and when left alone lapsed into a drowsy condition. When asked, he said that he had pain in the head, and pointed to the site of the wound. There was freer movement of both left arm and leg. The pupils were dilated. Temperature $100^{\circ}$; pulse 108 ; respiration 26 . $\mathrm{He}$ asked for the bed-pan when he wanted to pass a motion, but the urine was passed involuntarily. On the 19 th he was more conscious, answered when spoken to, but was still irritable, and complained of pain in the head. $\mathrm{He}$ was able on this day to raise the left arm above the head, but the grasp of the hand was weak. The movements of the left leg were much stronger, and almost equal to those of the right. There had been no recurrence of fits, and he was conscious of the desire to pass urine. Temperature $98^{\circ}$; pulse 100 ; respiration 20. On the 20th he was somewhat irritable, slept a great deal, but was much more sensible in every respect. On the 21st the irritability appeared to have disappeared, and he was less drowsy. The stitches were removed, but a small drainage-tube was retained in the wound. $\mathrm{He}$ continued to improve during the next two days, and the wound had a most healthy appearance, though giving issue to a good deal of healthy pus. On the 25 th there was a sharp rise of the temperature to $103 \cdot 8^{\circ}$, with a pulse of 140 , and he complained of pain in the head. When the bowels (which had not been open for two days) were relieved by a dose of castor oil, the temperature fell to $98^{\circ}$. On the 28 th the appearance of the wound, which had been satisfactory, gave rise to fear lest some hernia cerebri should threaten; the granulations were exuberant and fungating, and pulsation could be observed in them. This condition subsided with the free use of iodoform, and all the other symptoms improved, the only trace of the former paralysis being noticed in the weaker grasp of the left palm. During the next few weeks the cicatrisation became firm, and on Aug. lst the boy was walking about without any trace of paralysis, but with some slight exudation from some superficial granulations at the apex of the wound. He has remained perfectly well up to the present time (eleven months since the operation), and has no trace of weakness or loss of power in either extremity. There is a depression of the scalp at the site of the removed bone; but though pulsation is well marked, firm pressure on this region produces neither pain nor other symptom.

The case seems to present the following points of interest. 1. The distance of the position of the depressed bone from the site of the scalp wound. The absence of any lesion exposed by the wound in the scalp did not in the first instance justify its extension, and the subsequent symptoms were such as might justly be ascribed to such laceration of the brain as might be expected in a case of such severe injury.
It was fortunate that the paralysis which ensued was so dis. tinctly marked in its distribution as to point distinctly to compression of the motor area of the left arm and leg, and thus the necessity for exposing the surface of the brain in that region could not be questioned, so soon as the paralysis became evident. 2. With regard to the treatment of the abscess, the event has proved that no harm accrued from the freedom with which all granulation tissue which formed the margins of the cavity was removed, even at the expense of some healthy brain tissue. On the other hand, it may be doubted, if the contents of the cavity had been evacuated only, and the surface which surrounded them had not been removed, whether these granulations might not have become more active, and have given rise to hernia cerebri, besides involving more of the brain tissue that zurrounded them. As it was, the granulations at one time became so far exuberant as to give rise to fear lest a hernia cerebri should supervene. That these yielded to the free use of so powerful an antiseptic as iodoform points to the probable cause of this condition-viz., a septic condition of the granulations,and is indicative of a form of treatment which is much more likely to be successful than those forms of pressure which were formerly recommended. 3. The symptoms which followed the operation were not without peculiar interest. The fits that followed showed that a considerable amount of disturbance existed in the substance of both hemispheres, since the spasms were noted to effect the right side of the body and face as well as the left, although the power of movement which returned rapidly in the left arm and leg showed that the area which governs the functions of those parts regained its activity as soon as the pressure was removed.

Grosvenor-street, W.

\section{A CASE OF COMA DUE TO CEREBRAL ABSCESS;}

SUCCESSFUL EYACUATION OF THE ABSCESS BY THE TREPHINE ; DEATH.

By A. MARMADUKE SHEILD, M.B., F.R.C.S., ASSISTANT SURGEON, AND SURGEON IN CHARGE OF AURAL DEPART* MENT, CHARING-CROSS HOSPITAL.

THE majority of cases of cerebral abscess are secondary to caries and necrosis of the cranial bones, and especially to such affections of the petrous portion of the temporal bone The abscess will generally be found in the temporosphenoidal lobe, and more early in the middle lobe of the brain, or the cerebellum. These cases are usually associated with long-standing otorrhvea. They may also be complicated by meningitis and pyæmia, and thus render a difficult diagnosis still more enibarrassing. In most cases, inquiry will elicit that there have been premonitory symptoms. The pains spoken of by patients, too often by doctors, as "neuralgic," are, in truth, due to chronic basal meningitis, localised to the region over the inflamed and diseased bone. The symptoms of cerebral abscess are known to be dubious and misleading in the highest degree. When the collection of pus is found in the temporo-sphenoidal lobe, the symptoms are exceedingly vague. This portion of the brain has few certain motor functions. Post-mortem examination may reveal disease in it for the first time. In a remarkable paper by Macewen, ${ }^{1}$ two cases of abscess in the temporosphenoidal lobe, treated by operation, are related. The first case was unsuccessful. The patient was in extremis at the time of operation, and in this respect resembles the case I am about to narrate. At the necropsy, the whole lobe was found to be destroyed, and the neighbouring convolutions implicated in encephalitis. The second case was successful, and is one of the most interesting in a remarkable series, as indicating the possibility of localisation of abscess in the temporo-sphenoidal lobe by a careful process of exclusion conducted by a careful and competent observer. The details of the case about to be described are as follows.

A woman aged thirty-two was admitted into Charing. cross Hospital on Sept. 26th, 1888, in a condition of profound coma. The only history obtained of her case was to the effect that she had been under the care of a neighbouring 
The Lancet,] DR. F. DE HAVILLAND HALL: HAY FEVER AND HAY ASTHMA. [JunE 15, 1889.1183 practitioner for "pains in the head," that these had lately
got worse, and that since the morning she had been drowsy and stupid. On examining the right ear, pus was found in the meatus, and a foul odour was apparent on close investigation. The auditory canal being cleaned, the tympanic membrane was found to be quite destroyed, and protuberant granulations were abundant. The pupils were equal, somewhat dilated, not acting to light. There was no squinting, and optic neuritis was not looked for. The face was dusky and congested, the respiration slow and stertorous, the skin burning hot to the touch, and the temperature $104^{\circ}$. There were no evidences in the lungs, joints, or skin of general pyæmia; no tenderness or oedema in the mastoid region, or fulness down the course of the jugular vein. The pulse was regular and compressible, 120 to the minute. Free leeching was tried without benefit. The case was watched for about three hours. The coma was obviously deepening and the patient in a condition of impending death. About 6 P.M. I operated. The right side of the head was shaved and carefully cleansed with strong carbolic acid. A large flap, with its base downwards, was formed above and behind the auricle, the vessels being twisted. A large trephine was applied at a spot one inch above and a quarter of an inch behind the external auditory meatus. On removal of an exceedingly thin disc of bone the dura bulged strongly, did not pulsate, and no suppuration could be detected beneath it. A small trocar and cannula was now passed downwards and forwards into the substance of the temporo-sphenoidal lobe for about one inch. On withdrawing the trocar greenish pus exuded, and on enlarging the aperture with a director about half an ounce of very fetid pus escaped, mingled with brokendown cerebral débris. A tube was gently inserted into the abscess cavity, which was washed out with warm antiseptic lotions. The flap was replaced and carefully sutured, the tube being brought out through an aperture in its base, and the parts dressed antiseptically. The stertor was somewhat lessened by the operation, and slight movements of the limbs were afterwards noticed; but it may be generally waid that no marked improvement resulted. The woman died at 2 A.M. on the following day. A post-mortem examination was refused.

Notwithstanding the unsatisfactory termination of this case, I have thought right to record it. In such an important surgical proceeding as trephining for cerebral abscess, it is meet and right that all cases should be related. In the absence of a post-mortem examination, we can only conjecture the cause of the failure of the operation. Meningitis, encephalitis, or the bursting of the abscess into the ventricular cavities, are possible and probable explanations. The operation was, in fact, undertaken too late, the general paralysis indicating a condition that is too rarely relieved by the trephine in cases of pressure by pus or blood.

Mr. Barker, in a paper on the subject of Ear Disease and its Cerebral Complications, ${ }^{2}$ alludes to the comparative frequency of temporo-sphenoidal abscess, and the tolerance of that region of the brain to surgical interference. Treating of the regions affected, he speaks thus: "Nine-tenths of them (abscesses) will be found to lie within a circle with a radius of three quarters of an inch, whose centre lay one and a quarter inch above, and the same distance behind the auditory meatus." In the same paper it is pointed out, with truth, that subdural suppuration is of ten most difficult to certainly differentiate from abscess. But this, too, may be reached and relieved by operating in the same region, which is probably the best situation to choose. It cannot be too strongly impressed upon us all that the symptoms of cerebral abscess may be readily overlooked and misunderstood. It is only by the general symptoms of vomiting, delirium, optic neuritis, and the like that we may suspect abscess in the temporo-sphenoidal lobe; localisation symptoms are quite exceptional. Percussion of the skull might be of some use, and fixed pain, or oedenia of the soft parts, are valuable guides when present.

Stratford-place, W.

2 Ibid., June 11th, 1887.

LONGEVITY IN IRELAND.-Among the deaths recorded during the quarter ending March were nineteen centenarians. Seven were aged 100 years, one each at 101 , 102 , and 103, two at 104, one at 105, two at 107, one at 109, two at 110 , and one at 111 years respectively.

\section{HAY FEVER AND HAY ASTHMA.}

BY F. DE HAVILLAND HALL, M.D., F.R.C.P., PHYSICIAN TO OUT-PATIENTS AND IN CHARGE OF THE THROAT DEPARTMENT AT THE WESTMINSTER HOSPITAL.

THE return of the hay season and the prospect of there being an unusually abundant crop this year have induced me to direct attention to hay fever, which is a subject of ever-increasing interest, more especially as with advancing civilisation it appears to be gradually becoming more prevalent. Sir Andrew Clark struck a true note when, in the Cavendish Lecture ${ }^{l}$ he emphasised the doctrine that there are three great factors concerned in the evolution of hay fever: (1) A nervous constitution or idiosyncrasy, sometimes inherited and sometimes acquired; (2) a local condition of irritability, involving the nervous, vascular, and cellular constituents of the affected parts, and which, when excited, disturbs the chemical, morphological, and secretory changes taking place therein; (3) external, exciting, or determining causes-i. e., the agents which are capable of calling into action the irritability of the parts concerned. The first of these factors is so vague, and is so little susceptible of any direct treatment, that it will be out of place to discuss it at any length in a paper designed to be practical. As regards the third factor, there is an overwhelming weight of evidence in favour of the view that, in this country at all events, the most potent external exciting cause is the pollen of various grasses. Still, there are cases in which other irritants, such as ipecacuan, dust, or even touching certain zones of the mucous membrane of the nose, will induce a train of symptoms indistinguishable from hay fever, and, as is well known, a bright light or great heat will oftentimes start an attack in persons subject to the affection. This brings me to the consideration of the second factor, the condition of irritability of the nasal mucous membrane. In spite of what has been done in America and in Germany, the interior of the nose has received too little attention in the treatment of hay fever. Notwithstanding the extreme views to which his enthusiasm led him, there can be no doubt that Hack did good service to medicine by pointing out that changes in the interior of the nose play a prominent rôle in the production of symptoms in other organs, as examples of which may be cited cough, migraine, supra-orbital neuralgia, giddiness, redness and swelling of the nose, \&c.; but it is in hay fever, asthma, and paroxysmal sneezing that we meet with the most pronounced examples of these reflex neuroses. The changes met with in the nose in cases of hay fever are usually of a hypertrophic character-i. e., they constitute the condition known as hypertrophic rhinitis; or they may may be more limited, consisting only of a puffy swelling of the inferior turbinated bodies, or of an enlargement of the posterior extremities of the same; in some cases the middle turbinated bodies are chiefly affected, or one or more polypi may be present, or there may be some abnormal condition of the septum. In a case seen last autumn, a gentleman who had been a great sufferer for many years from hay fever ceased to be troubled as soon as a perforation in the septum occurred, thus apparently proving that the irritable zone from which the reflex started was situated on the septum. Be the local changes what they may, the paroxysm of hay fever is invariably accompanied by swelling and engorgement of the erectile tissue, which forms so important an element of the inferior turbinated body, but which is also present in other parts of the nasal mucous membrane; and following this there is an increased secretion, which is at first purely serous, but which may become muco-purulent.

In treating a case of hay fever, the three factors concerned in the production of the disease must be borne in mind. It will, therefore, be desirable in the first place to improve the general health of the individual as far as possible. There can be no doubt that in many cases the injudicious use of alcohol aggravates the severity of the disease. The depression and exhaustion produced by hay fever are oftentimes so great that the patient has recourse to stimulants to counteract these effects; but though he may experience temporary relief, in the end alcohol in any quantity only aggravates the local trouble, to say nothing of the more remote evils that may result from its use. Sir Morell

1 Brit. Med. Jour., June 11th, 1887. 